\title{
Producing micron- and nano- size formulations for functional foods applications
}

\author{
Thomai Panagiotou' ${ }^{1}$ and Robert J. Fisher ${ }^{2}$
}

${ }^{1}$ Microfluidics International, IDEX Material Processing Technologies, 30 Ossipee Rd, Newton, MA 02464, USA; ${ }^{2}$ Massachusetts Institute of Technology, Chemical Engineering Department, Bldg 66, Rm 305, 77 Massachusetts Avenue, Cambridge, MA 02139-4307, USA

Corresponding Author: Thomai Panagiotou, Microfluidics International, IDEX Material Processing Technologies, 30 Ossipee Rd, Newton, MA 02464, USA

Submission date: April 29, 2013; Acceptance date: July 3, 2013; Publication date: July 8, 2013

\begin{abstract}
:
Background: Nutrient deficiencies affect the health and wellness of large populations around the world. For example, the majority suffer from vitamin, essential fatty acid (such as omega-3), dietary fiber, and other important ingredient deficiencies due to their limited supply in the human food chain. Current trends in the nutraceutics industry to place these substances in higher, moreefficiently dispersed quantities in our food have become critically essential to their business plans. Nutrients in the form of small solids or droplets improve bioavailability. However, there remain numerous barriers to successful implementation of cost effective manufacturing processes. These challenges are addressed in the work presented here with particular focus on stability, bioavailability, and consumer acceptance. The goal is to develop large scale manufacturing systems that implement efficient platform technologies, with their respective operational maps, to produce functional food formulations, with particle sizes of these specially formulated nutraceutical ingredients in the micron-and nano- range.
\end{abstract}

Objective: Demonstrating that stable micron- and nano-size emulsions, liposomes, and aqueous suspensions of functional food formulations can be produced using both "top down" and "bottom up" methods is our main objective. Addressing the challenges associated with the incorporation of these ingredients into large scale manufacturing systems, mainly mechanical stability and related shelf-life issues, is also a focus. That is, to develop proper processing protocols providing improved quality foods enriched with ingredients that are in limited supply in our food chain; to enhance human health and wellness world-wide.

Methods: The formulations considered here typical of those used for increasing bioavailability of the infused, specially formulated ingredients with anti-cancer, anti-aging, and in-general wellness properties, lowering fat content and enhancing the shelf-life stability. Included are (a) 
an oil-in-water (fish oil/omega-3) emulsion, (b) liposome chaperones to vitamin $\mathrm{C}$, and (c) aqueous suspensions (curcumin crystals, lutein/carotenoids, and fiber in soy milk). The production techniques include both "top-down" particle size reduction and "bottom-up" formation of crystals/precipitates via solubility adjustments. Both techniques are based on high shear processing of multiple liquid feeds. Using an impinging jet system, micro-mixing scales less than $100 \mathrm{~nm}$ were obtained.

Results: (a) All nano-emulsion types, single, double and larger, either as oil-in-water and waterin-oil, can effectively be produced from various formulations using "top-down" methods. Illustrated here are single, oil-in-water systems; concentrations of 12-14 wt. \% fish oil/omega-3 were mixed with water containing food grade surfactants. The high shear processing produced stable, submicron particles; with median particle sizes of 119-163 nm, no particles larger than 1 micron, and the "fish" odor was suppressed. Pertinent discussions related to the other types are also given as suggested path forward approaches for the development of nutrient enriched functional foods. This includes water-in-oil formulations for reduced fat content and the delivery of multiple species via double and triple emulsions, as compared to liposome configurations.

(b) Although liposomes may be used to encapsulate both hydrophobic and hydrophilic substances, we selected liposomal vitamin $\mathrm{C}$ as our initial proof-of-concept system since it is absorbed into the body over four times more easily than its non-encapsulated form. After top down processing, the median size was $200 \mathrm{~nm}$, compared to a median size of about 5 microns obtained by traditional self-assembly protocols. (b) Aqueous suspensions of micron- and nanosize formulations were also accomplished. The top down size reduction technique was used for processing soy bean fibers and lutein and the bottom-up method used for curcumin crystals. The fibers initially had a median size of 150 microns and a bi-modal distribution was obtained after processing; $99 \%$ of the particles were smaller than 15 microns with median sizes at 10 microns and the larger peak at about $200 \mathrm{~nm}$. The curcumin submicron particles were formed via antisolvent crystallization; with stable particles in the range of $300-500 \mathrm{~nm}$.

Conclusions: Our study demonstrates that stable micron- and nano-size emulsions, liposomes, and aqueous suspensions can be produced using both "top down" and "bottom up" methods. The formulation properties, in terms of particle size and stability, strongly depend on the processing parameters used in terms of energy input and temperature history. The energy requirements of the "bottom up" methods may be substantially lower than those of "top down" methods. Although some of the processes presented here have been scaled up to commercial levels, more work is needed in terms of fully assessing the bioavailability of the produced formulations and optimizing the processes to minimize cost.

Key words: nano-emulsion, nano-suspension, high-shear processing, crystallization, curcumin, fish oil, liposomal vitamins: $\mathrm{C}$ and $\mathrm{E}$, lutein, nutraceuticals, omega-3, soybean fiber. 


\section{BACKGROUND:}

It is well documented that large populations around the world suffer from nutrient deficiencies $[1,2]$. As an example, the majority of Americans suffer from vitamin and essential fatty acid (such as omega-3) deficiencies. Consequently, to enhance human health and wellness globally, there exists a need for improved quality foods, enriched with ingredients that are in limited supply in our food chain. Many of these important ingredients have been removed from our natural food sources through selective breeding of plants, or during processing. Ideally, the human diet should consist of more low fat foods, rich in dietary fiber and contain specially formulated ingredients with anti-cancer, anti-aging, etc. properties with increased bioavailability.

Current trends in the nutraceutics industry to place these substances in higher, moreefficiently dispersed quantities in our food have become critically essential to their business plans. Some examples of current nutraceutical and functional foods trends include:

- Omega-3's for heart health, infant developmental needs, enhanced wellness

- Lowered fat content in food products for weight loss/control, overall health

- Higher fiber content in snack/nutrient bars and beverages for digestive health, weight control

- Plant sterols suspended in beverages for heart health, lowering cholesterol, anti-cancer benefits

- Phyto-specific poly-phenols and carotenoids to provide specific benefits, such as eye health

- Peptides for overall health/wellness

- Pro-biotics for digestive health and anti-cancer benefits, and

- Natural foods/minimally processed with no additives and/or no potentially toxic substances

- Particle size plays a major role in the ability to effectively accomplish these enrichment processes:

- Submicron particles help improve bioavailability and stability of formulations [3-10]

- Micron/nano-encapsulation can protect active ingredients, such as omega-3 oils from oxidation and also undesired taste and odor

Solid particles less than 20 microns can be incorporated into foods without substantial changes in texture, as in soy milk and yogurt, and virtually undetectable in the mouth; and Water droplets encapsulated in fat, e.g., solidified water-in-oil emulsions ("fat flakes"), reducing the quantity needed in foods requiring fats in their preparation, thus lowering caloric values.

Despite success with advanced delivery and transport technologies in the areas of pharmaceutics[3-9], barriers to success in the area of nutraceutics remain. These include long shelf-life stability requirements, oxidation protection, odor and taste control, mouth feel, and efficiency of delivery (which is directly tied to stability and bioavailability), as well as meeting requirements by regulatory bodies such as the US FDA. Proper processing of these nutraceutical and functional food formulations can help in overcoming many of these challenges. The work presented here addresses methods to accomplish this. 


\section{INTRODUCTION:}

The results obtained in our study and reported here demonstrate the effective use of high shear processing platform technologies that are suitable for the production of ingredients used in functional foods with micron- micro and nano- size specifications. This includes emulsions, suspensions of solids, and cargo loaded vesicles prepared via specifically developed operational maps. The formulations selected for proof-of-concept studies provide evidence that successful implementation of large scale, cost effective manufacturing systems will emerge utilizing these methodologies. Furthermore, the active ingredients in these formulations represent a broad spectrum of material processing requirements, as well as their inherent nutritional, health and wellness attributes. The following paragraphs contain a few brief comments in reference to some of these beneficial characteristics.

Emulsions: The food industry has used emulsions at the micron size extensively in the past. This includes oil/water $(\mathrm{o} / \mathrm{w})$, water/oil (w/o), single, double and triple emulsions for their ability to encapsulate hydro- and lipo- philic species simultaneously [11]. Figure 1 shows schematics of $\mathrm{o} / \mathrm{w}$ and w/o single emulsions. Nano-emulsions, stabilized with functionalized surfactants have shown great potential, with ongoing research and modeling efforts that successfully predict size and performance are evolving into the commercialization stage [10, 12].

Entrapment of sub-micron scale cargo-loaded emulsions into macro-scale food matrices has exhibited great potential as a delivery vehicle with controlled release capabilities, however optimization remains unrealized. There exists at significant relationship between the entrapping process and emulsion formulation, shelf-life and system quality evaluation. Reported previously are methods used to improve product quality by enhancing the emulsion formation steps [10]. Detailed experimental protocols, theoretical explanations, and path forward recommendations to overcome challenges and meet expectations in these emerging opportunities have been presented elsewhere in the literature [10-12]. Selected here to illustrate successful use of those platform technologies (as developed earlier) for sub-micron sizing studies is a single emulsion systems of particular interest for incorporation into macro-scale products for commercialization; omega-3's in water $(\mathrm{o} / \mathrm{w})$.

The selection of generic fish oil as one of the key ingredients to be studied here is also based upon a general consensus in the food industry to add omega-3, i.e., polysaturated fatty acid docosahexanoeic acid (DHA) and eicosapentanoeic acid (EPA), into long shelf-life food categories, such as cereal. However, due to its high degree of unsaturation, autoxidation is a particularly undesirable event. This is especially true for these long chain fatty acids (DHA and EPA). During the oxidation process, volatile secondary lipid oxidation compounds are formed. Many of these compounds have a very low odor threshold, leading to development of off-flavors, and thus a decreased shelf-life. In response to the sensitivity and easily oxidizable property of omega-3, encapsulation is required to protect these fatty acids from autoxidation and thus prevent off-flavor and improve consumer acceptance. With the aims of achieving more effective encapsulation and driving down DHA/EPA cost per serving, optimized encapsulation technology is currently undergoing substantial research efforts [16-22]. 

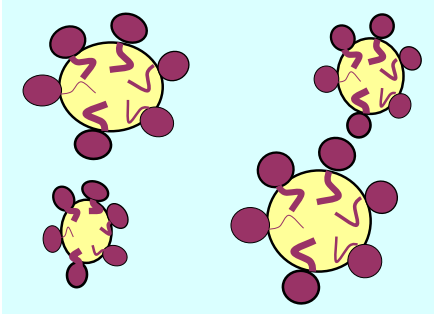

(a)
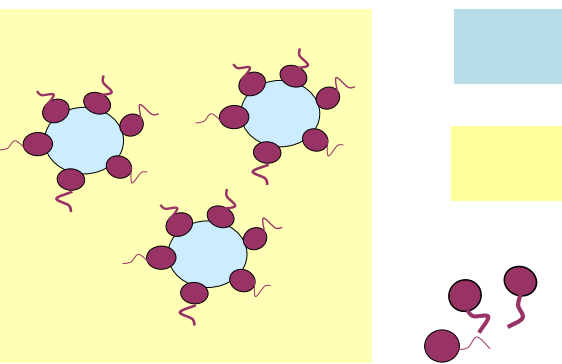

Water Phase

Oil Phase

Surfactant

molecules

(b)

Figure 1. Schematic of (a) oil/water (o/w) emulsion, and (b) water/oil (w/o) emulsion.

The water in soybean oil emulsion (w/o) was selected due to the interest in fat reduction in spreads and doughs. i.e., lower fat content in food products for weight loss/control, and overall health. The idea is to produce hydrated "fat flakes", through solidification of the emulsion, for use in processing and /or in content of consumer macro-scale products, primarily dough based items. This is desirable from both a consumer health and corporate financial perspective. Unfortunately, many challenges exist. However, some can be overcome through proper formulation and processing techniques, as are discussed in this work. One major concern is the stability of these flakes; in their (and/or the product) shelf-life and during the process of incorporating them into these macro-products. For example, it is highly undesirable for the flakes to smear and leak water. Clearly, high water content is sought, but must be balanced with respect to mechanical, thermal, and rheological properties.

Liposomal vesicles: A liposome is a synthesized lipid vesicle with the ability to encapsulate both hydrophilic and lipophilic species simultaneously for the administration of nutrients and pharmaceutical agents [13,23], see Figure 2. This characteristic is a result of their composite structure, made of phospholipids and small amount of other molecules, such as targeting ligands attached to their surfaces. Generally, liposomes are formed by hydrating the dry lipid film/cake in an agitated aqueous medium. These sheets self-close forming large, multi-lamellar vesicles (LMV) preventing the interaction of water with the hydrophobic region of the newly formed bilayers. Once a stable LMV suspension has been prepared it can be sized reduced by a variety of techniques; i.e., energy input via sonication or mechanical based methods to develop high shear fields, such as in extrusion, high pressure homogenization, or impinging jet technologies [10-12].

Until recently, liposomes were used primarily for targeted drug delivery [3-9, 13]. Their versatility is now being implemented for the specific oral delivery of dietary and nutritional supplements $[10,24,25]$ to improve their bioefficacy since they had exhibited low absorption and bioavailability rates when administered via traditional tablet and capsule methods. Furthermore, encapsulation of nutrients within liposomes provides a very effective method of bypassing the destructive nature of the gastric system, and when appropriate, avoids detection by the immune system, i.e., cells of the reticulo-endothelial system (RES) [23, 26, 27]. 

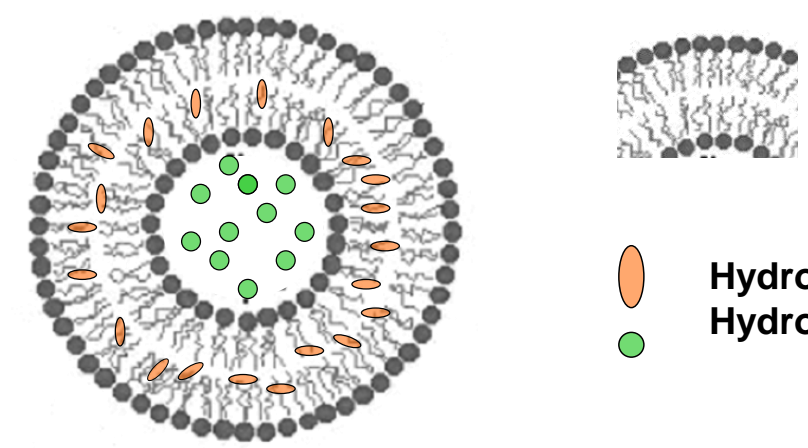

Hydrophobic compound Hydrophilic compound

Figure 2. Schematic of a liposome vecicle used for encapsulation of hydrophobic and hydrophilic compounds.

Specific targeting is possible by functionalizing via surface ligands such as vitamins, monoclonal antibodies, and specific antigens [23]. In this manner, lower dosages of the active nutrient for delivery to specific cells/organs can be used to accomplish higher bioavailability than previously obtained. Not only is cost impacted, but possible toxicity to other cells types is minimized. It should also be noted here that many of the same features associated with the use of liposomes can be obtained using double and triple emulsions [10,11], as mentioned

Fibers: The inclusion of micron- and nano-fibers into macro-foods, such as soy milk and yogurt, has not only the beneficial effects for digestive health, and weight loss/control, but can also provide chaperone capabilities. The small size is required if mouth feel and/or high surface area for enhanced adsorption characteristics are sought. Poorly soluble nutrients can be delivered exploiting the high adsorption characteristics of various natural fibers obtained from fruit and vegetable sources. Furthermore, undesired taste/odor associated with these nutrients, such as fish oils, can be masked. This chaperone capability of fibers has also been promoted as a means of toxin removal, for example, from either contaminates ingested and/or hormonal overexpression.

Lutein: One of 600 known naturally occurring carotenoids, lutein (a xanthophyll) is a lipophilic molecule and thus is essentially insoluble in aqueous media. It is synthesized only in plants and large quantities are found in green leafy vegetables such as spinach and kale. Although lutein is also found in egg yolks and animal fats, it is only there because of its presence in the dietary plants consumed for these secondary sources. Within green plants, xanthophylls serve to modulate light energy and as a non-photochemical quenching agent. Animals utilize lutein as an antioxidant and to adsorb blue light [28]. The human retina accumulates lutein (in the macula area, responsible for central vision) and may serve there as a photo-protectant from the free radicals produced by the high energy photons of blue light [29, 30]. The light-absorbing properties can be attributed to the presence of the polyene chain (long chromophore of conjugated double bonds). Unfortunately, the polyene chain is chemically unstable in acids and susceptible to oxidative degradation by light or heat. 
A direct relationship between lutein uptake and pigmentation in the eye has been confirmed through numerous research studies [29-32]. They also showed that an increase in macula pigmentation decreases the risk for eye diseases; in particular, age-related macular degeneration (AMD), a blindness affecting 1.2 million Americans. Lutein supplements are also correlated with reduced incidence of nuclear lens opacities; cataract development [33].

Curcumin(Curcuma longa L.): Curcumin, a polyphenol derived from the powdered rhizomes of turmeric [34], has been popularized by its use as both a spice (found in turmeric) and a coloring agent (phenol yellow) in a number of foods, drugs and cosmetics. However, its value as a powerful anti-oxidant remains largely unexploited. Studies have illustrated great potential for this polyphenol derivative as both an anti-inflammatory and an anti-carcinogenic, as well as significant therapeutic value in the treatment of various chronic diseases [35-39]. Unfortunately, curcumin's hydrophobic nature, and thus poor water solubility, oral bioavailability and photo stability [40, 41, and 44] have proven inhibitors in its application for such uses. It is generally accepted that this limited solubility, combined with extensive systemic metabolism and clearance rates, is likely responsible for the low bioavailability of curcumin after oral delivery [42, 43]. Despite these drawbacks, the inherent positives of curcumin as a health/wellness agent have yielded a resurging effort in reformulation protocols [42, 45-48]. When administered orally, it has been observed that only trace amounts of the curcumin appeared in the blood (compared to direct absorption by the gastro-intestinal tract [40, 49-51], where most was excreted in the feces and urine after rapid uptake by metabolization and clearance processes. It has been deduced that in order to obtain maximum in vivo efficacy, the release rate of curcumin from its delivery vehicle must be controlled at a moderate flow, i.e. it is best matched with the various uptake and clearance rates; therefore formulation design is crucial.

Research efforts focused on comparing potential efficacious formulations of curcumin identified a nano-crystal solid dispersion (CSD) as the most promising formulation [41]. Researchers concluded that on the basis of their overall observations, considering surface morphology, size distribution, crystallinity, thermal properties and dissolution rate, a strategy utilizing CSD would be most efficacious in enhancing in vivo performance and photo chemical stability. A significant improvement in pharmacokinetic behavior (with respect to oral bioavailability) was observed; i.e., an increase of 16 fold., Following-up on the CSD success, processing strategies to prepare stable nano-crystal formulations were investigated, both from a top down [40] and bottom up [52] perspective. The top down group [40] used high-pressure homogenization to reduce pre-milled aqueous suspensions (micrometer sized particles) to a plateau value of approximately $600 \mathrm{~nm}$. This was accomplished only after the tenth pass through the homogenizer; thus proving highly energy intensive. The bottom up group [52] attempted to form nano-crystals via the solvent /anti-solvent technique (curcumin/ethanol and water) in a low pressure micromixer. Their product was amorphous nano-spheres (less than $100 \mathrm{~nm}$ ) as the initial precipitate, which then went through aggregation/recrystallization before transforming into needle-shaped crystals with lateral dimensions of approximately $200 \mathrm{~nm}$ and micrometer scale lengths.

Clearly, an alternative bottom-up approach that produces the desired crystal size and morphology directly without further downstream processing would be much more efficient and 
possibly yield smaller sized particles with a narrow size distribution [7]. The many benefits associated with a true bottom-up strategy have been well documented $[6,7]$ and success with that strategy is what formed the basis for our approach as taken here. Fortunately, this protocol also proved successful as substantiated by the results that are presented here.

\section{METHODS:}

The formulations studied, with results reported here, include: (a) oil-in-water (fish oil/omega-3) emulsions, (b) liposome chaperones to deliver vitamin $\mathrm{C}$, and (c) aqueous suspensions (fiber in soy milk, lutein/carotenoids, and curcumin crystals). When appropriate, either food grade, or specifically designed proprietary surfactants were used for size/stability control and/or reduced energy input.

Material size characterization (droplet or solid particle) was by mean diameter and polydispersity index (PDI), indicating size distribution. Dynamic and static laser light scattering were used for particle size analysis, DLS and SLC, respectively. For DLS, a Nano-S from Malvern Instruments, Inc, Southborough, MA was used, while for SLC a Horiba 910 instrument was used, with measuring capabilities 0.6- $6000 \mathrm{~nm}$, and 20nm-1000 microns, respectively.

The production techniques are both "top-down" particle/droplet size reduction and "bottom-up" formation of solid particles via solubility adjustments to yield crystals /precipitates. Both techniques are based on intense mixing accomplished through high shear processing of multiple liquids. At the core of this high shear fluid processing technology lays an impinging jet chamber, containing opposing micro-channels which can produce jet velocities that approach $500 \mathrm{~m} / \mathrm{s}$. The high shear and turbulence generated result in the obtainment of micro-mixing scales that are less than $100 \mathrm{~nm}$. Descriptions of system components/devices and associated operational protocols for the various process configurations that constitute our platform technology are available in prior publications $[10,53]$.

The outcome is either (a) particle size reduction during the "top down" processing, or (b) mixing of reactants to produce nano-crystals at scales below $50 \mathrm{~nm}$ via a "bottom up" process (such as crystallization, precipitation or chemical reactions [6-13, 53]). Both operational protocols as employed here, via our platform technology, include two or more continuous streams that interact within the impinging jet chamber. Due to the magnitude of the micromixing scales obtained, the transport and reaction processes are rapid and near completion, and thus the size of the resulting particles can be controlled to be within that size range. The specific operational map developed for each individual formulation was therefore based on the unique physical and chemical characteristics of that formulation. This strategy generated the appropriate response surfaces required to determine the optimum processing conditions for a given objective function; based on product quality specifications. Thus, the dimensions of the micro-channels within the impinging jet devices and energy input (inlet pressure and temperature), establishing the fluid velocity, degree of turbulence (energy dissipation rate), residence time, and temperature history during processing, were varied to obtain the optimized performance.

\section{RESULTS AND DISCUSSION:}

(a) The nano-emulsions were produced from two formulations using "top-down" methods. These oil-in-water systems were prepared using fish oil for the omega-3 nutrient source. Fish oil 
at concentrations of 12 and 14 wt. \% was mixed with water containing food grade surfactants, namely gelatin and whey protein, respectively. Initially, the oil formed droplets of varying sizes, up to 30 microns in diameter. These macro-emulsions were unstable and had a strong fish odor. The materials were processed with a Microfluidizer processor at the conditions shown in Table 1.

Table 1. Processing conditions of two fish-oil o-w emulsions

\begin{tabular}{|c|c|c|}
\hline Formulations & $\begin{array}{l}\text { Processing pressure/ } \\
\text { Number of Passes/ } \\
\text { Interaction chambers }\end{array}$ & $\begin{array}{l}\text { Process Temperature/ } \\
\text { Cooling }\end{array}$ \\
\hline $\begin{array}{l}\text { Sample } 1 . \\
12 \% \text { fish oil - gelatin }\end{array}$ & $\begin{array}{l}1500 \mathrm{bar} / 4 \text { passes/ } \\
\mathrm{F} 12 \mathrm{Y} *_{-} \mathrm{H} 30 \mathrm{Z}(75 / 200 \mu \mathrm{m})\end{array}$ & $\begin{array}{l}50^{\circ} \mathrm{C} \\
\text { No cooling }\end{array}$ \\
\hline $\begin{array}{l}\text { Sample } 2 . \\
14 \% \text { fish oil }- \text { whey } \\
\text { protein }\end{array}$ & $\begin{array}{l}1500 \mathrm{bar} / 5 \text { passes } \\
\mathrm{F} 12 \mathrm{Y}^{*}-\mathrm{H} 30 \mathrm{Z}(75 / 200 \mu \mathrm{m})\end{array}$ & $\begin{array}{l}20^{\circ} \mathrm{C} \\
\text { Cooling after each pass }\end{array}$ \\
\hline
\end{tabular}

*Note: The F12Y is the highest shear 75 micron chamber.

After processing, the formulations had submicron particles, with median particle size 119-163 $\mathrm{nm}$ and no particles larger than 1 micron. Figure 3 depicts microscope pictures of unprocessed and processed gelatin emulsion, as well as the particle size distributions of the two emulsions after processing. The formulations were stable for several weeks at ambient temperature, the fish odor was suppressed, and the oil was protected from oxidation.

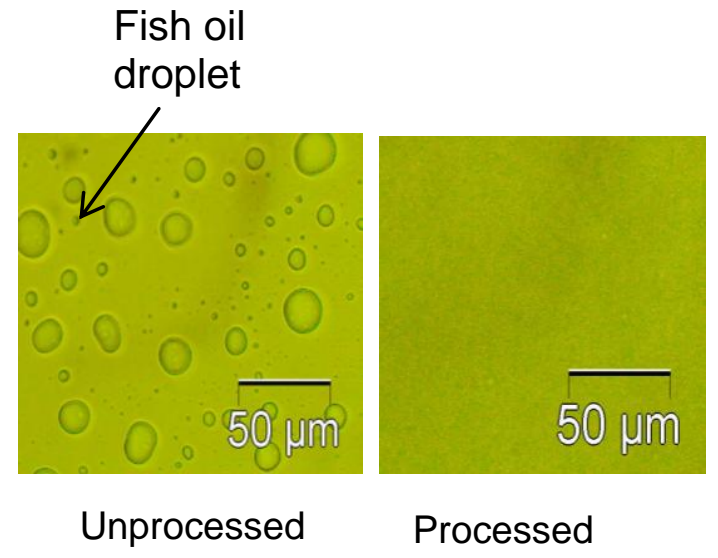

(a)

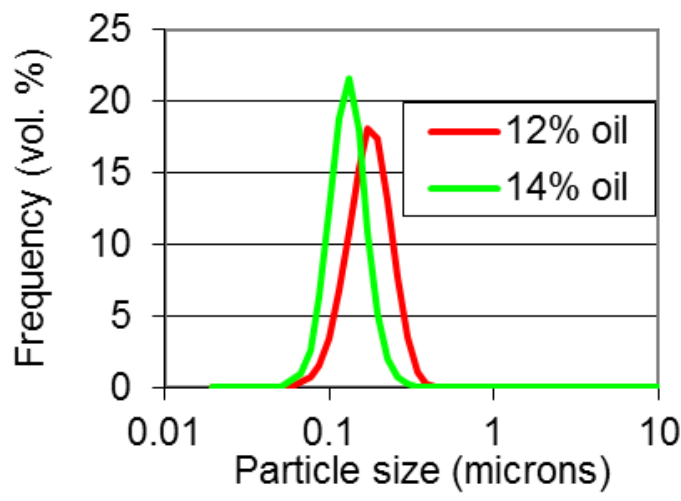

(b)

Figure 3. (a) Microscope pictures of unprocessed and processed gelatin emulsion, and (b) particle size distributions of the two emulsions after processing. The median particle sizes were 119 microns for the $12 \%$ and 163 microns for the $14 \%$ emulsion.

The temperature history during processing, as well as the energy input, (which determines the mixing intensity and energy density transfer rate), were critical in achieving the desired product 
quality, such as droplet size and stability. The importance of temperature is its effect on viscosity ratios, surfactant adsorption rate on droplet surfaces, collision frequency, and input jet momentum.

(b) Although liposomes may be used to encapsulate both hydrophobic and hydrophilic substances, we selected liposomal vitamin $\mathrm{C}$ as our initial proof-of-concept system since it is absorbed into the body over four times more easily than its non-encapsulated form. Initial encapsulation by traditional self-assembly protocols yields a median size of about 5 microns. After top down processing, the median size was $200 \mathrm{~nm}$.

The effect of the number of passes was investigated, see Table 2 . It can be seen that initially the particle size decreases with the number of passes. It then reaches a minimum at 4 passes. Subsequent passes initially increase the particle size and then slowly decrease it. This behavior has been also observed in the top down processing of emulsions. The explanation given is the presence of competing mechanisms of droplet size reduction and coalescence during processing.

Table 2. Processing conditions of a liposomal C formulation

\begin{tabular}{|c|c|c|c|c|c|}
\hline \multirow{2}{*}{$\begin{array}{l}\text { Processing Pressure/ } \\
\text { Interaction Chambers }\end{array}$} & \multirow{2}{*}{$\begin{array}{l}\text { Liposomal } \\
\text { vitamin C }\end{array}$} & \multicolumn{3}{|c|}{ Particle size (microns) } & \multirow[b]{2}{*}{ Average } \\
\hline & & d10 & d50 & d99 & \\
\hline - & Unprocessed & 1.707 & 4.393 & 12.440 & 4.797 \\
\hline \multirow{10}{*}{$\begin{array}{l}1400 \text { bar } \\
\text { F20Y *-H30Z } \\
(75-200 \mu \mathrm{m})\end{array}$} & \#1 pass & 0.304 & 0.458 & 0.863 & 0.470 \\
\hline & \#2 pass & 0.213 & 0.329 & 0.747 & 0.347 \\
\hline & \#3 pass & 0.202 & 0.287 & 0.508 & 0.296 \\
\hline & \#4 pass & 0.134 & 0.195 & 0.417 & 0.205 \\
\hline & \#5 pass & 0.266 & 0.366 & 0.593 & 0.370 \\
\hline & \#6 pass & 0.201 & 0.279 & 0.477 & 0.286 \\
\hline & \#7 pass & 0.252 & 0.333 & 0.544 & 0.338 \\
\hline & \#8 pass & 0.198 & 0.285 & 0.567 & 0.297 \\
\hline & \#9 pass & 0.152 & 0.206 & 2.893 & 0.261 \\
\hline & \#20 pass & 0.201 & 0.270 & 0.481 & 0.279 \\
\hline
\end{tabular}

*Note: The F20Y chamber has 20\% lower shear rate than the F12Y chamber shown in Table 1.

(c) Aqueous suspensions of micron- and nano-size formulations were also accomplished. The top down size reduction technique was used for processing soy bean fibers and lutein and the bottom-up method used for curcumin crystals.

Fiber containing soy milk: Currently, most soy milk formulations do not contain fiber from soy beans because of the large fiber size. Using our platform technology based processor, the fibers can be downsized and incorporated into the formulation and not affect consumer 
acceptance due to any undesired mouth feel issues, see Figure 4. The fibers initially had a median size of 150 microns and after processing, 99\% of the particles were smaller than 15 microns with a bi-modal distribution; median sizes at 10 microns and the larger peak at about $200 \mathrm{~nm}$. The small peak possibly corresponds to the droplet size of an emulsion formed from the oils in the soy milk and the water.

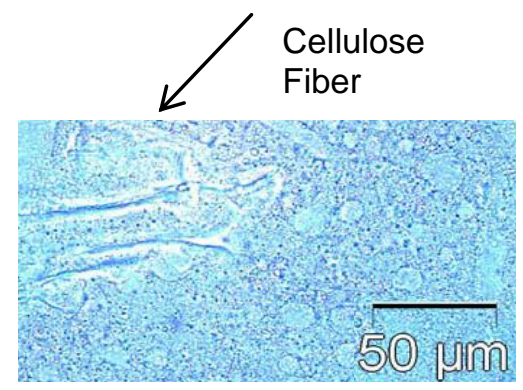

Unprocessed

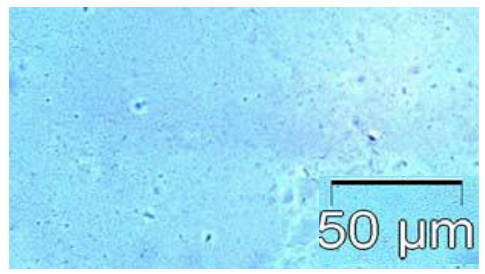

Processed

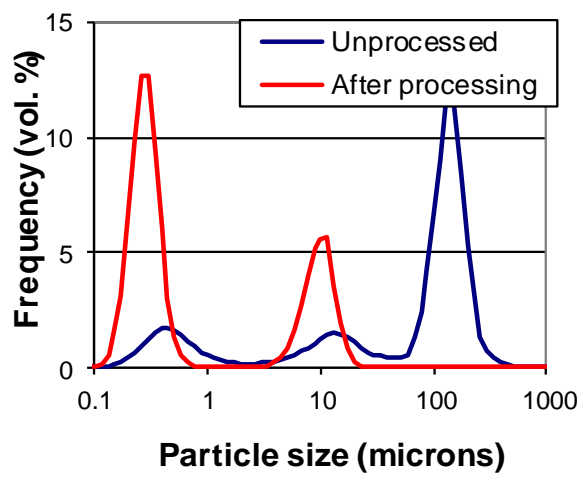

(b)

Figure 4. (a) Microscope pictures of unprocessed and processed fiber containing soy milk; long cellulose fibers can be seen in the unprocessed milk, and (b) particle size distributions of unprocessed and processed fiber containing soy milk; the processed milk does not contain any particles over 15 microns.

Lutein aqueous suspension: Although lutein is a lipid soluble oxygenated carotenoid, it can be stably suspended in an aqueous media if its particle size is in the sub-micron range, which also increases its bioavailability. Starting with mean particle sizes close to 100 microns, our downsizing process reduced that to less than $300 \mathrm{~nm}$; as seen in Figure 5.

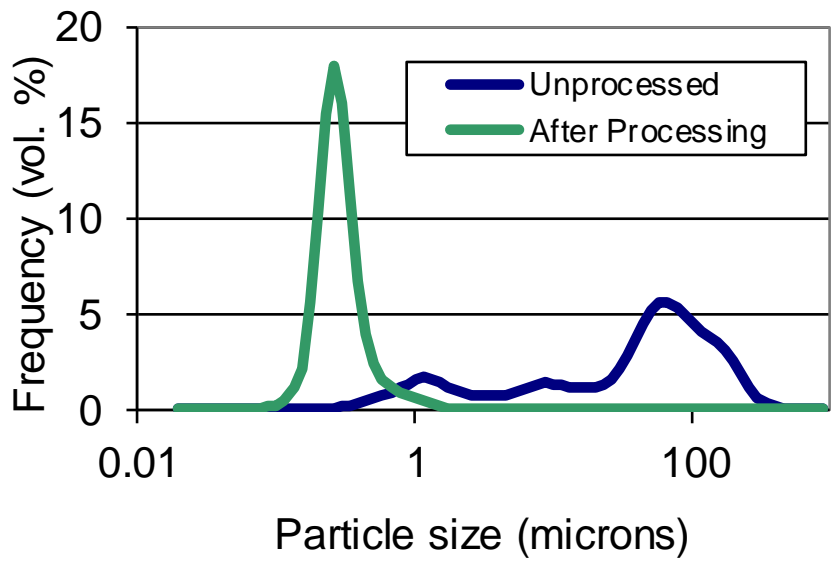

Figure 5. Particle size distributions of a lutein suspension before and after processing. The median particle size after processing was 0.273 microns. 
Curcumin nanosuspension: Curcumin submicron particles were formed via anti-solvent crystallization using the "bottom up" method. Various batches of stable submicron particles were produced directly as crystals. The particles were rod shaped, 100-200 nm wide and 300-600 nm long, see Figure 6 . The particles were stable in contrast to those produced by a low energy impinging jet technique, as reported previously by another research group [52]. The processing conditions are shown in Table 3.

Table 3. Production conditions for curcumin crystallization

\begin{tabular}{|l|l|}
\hline \multicolumn{1}{|c|}{ Formulation } & \multicolumn{1}{|c|}{ Processing Conditions } \\
\hline Solvent: DMSO & $\begin{array}{l}\text { Processor: CR5 (PureNano }{ }^{\mathrm{TM}} \text { ) } \\
\text { Anti-solvent: water }\end{array}$ \\
$\begin{array}{l}\text { Processing pressure: } 1500 \mathrm{bar} \\
\text { Surfumin conc. in DMSO: } 5-80 \mathrm{mg} / \mathrm{ml} \\
\text { Number of Passes: } 3-5 \text { passes* }\end{array}$ \\
$0.1 \mathrm{wt} \%$ & $\begin{array}{l}\text { Interaction chambers: F20Y-H30Z (75/200 } \\
\mu \mathrm{m})\end{array}$ \\
& $*$ The solvent stream was added to the anti- \\
& solvent stream gradually over 3-5 passes \\
\hline
\end{tabular}

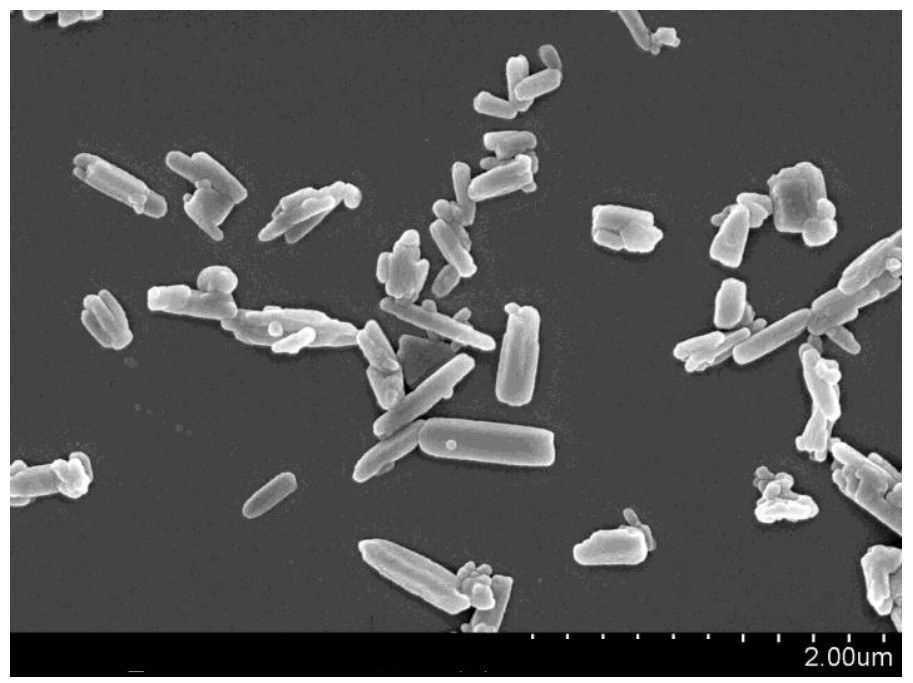

Figure 6. SEM picture of curcumin, crystallized using PureNano ${ }^{\mathrm{TM}}$.

The particle size depends strongly on the solvent-anti-solvent system used, super-saturation and the presence of surfactant. In comparing this "bottom up" curcumin crystallization method with the "top down" method used to produce submicron curcumin crystals by size reduction from large "preformed" crystals, it was found that the energy requirement of the "bottom up" method was a fraction of that required for the "top down" method. Furthermore, prior solvent/antisolvent crystallization studies [7] where particles sizes in the range $100 \mathrm{~nm}$ were obtained support the notion that since this is a bottom-up process it is possible to terminate growth at a 
smaller particle size. Thus, further optimization of the processing parameters should yield even better performance.

In commercializing such processes, there are several factors that should be considered: (a) bioavailability increase, (b) cost, and (c) incorporating the nano- and micron-size materials into foods.

Although there is substantial amount of work in pharmaceutics that correlates the particle size of the active ingredients with their bioavailability, the same cannot be said for nutraceuticals and foods. Determination of the bioavailability of active ingredients as a function of the particle size and formulation type is imperative.

The processes described in this work, namely production of nanoemulsions, fine suspensinsion and liposomes using Microfluidics technology are being used routinely for the commercial production of pharmaceuticals, chemicals, cosmetics, etc. In the food and mutraceutical areas, the commercial scale production has started in recent years. Fish oil emulsions, vitamin formulations and food flavoring and coloring formulations are currently produced at commercial_scales using Microfluidizer processors.

In commercializing such processes, the cost has to be minimized. A major factor contributing to the operational cost is the electrical energy demand of the process, which is proportional to the process pressure, the number of passes and the mass of material produced. In a separate study, we are specifically looking at ways to minimize cost by optimizing simultaneously the process parameters and the hardware (interaction chamber designs) for a specific formulation.

For many applications, the micron- and nano- formulations described here will have to be incorporated in foods, including doughs, cereals and energy bars, beverages, etc. For such applications, these formulations may need to be specially designed so they retain their stability within the food matrix. Critical parameters affecting the incorporation of micron- and nanoformulations into solid food matrices are discussed in ref. 10.

\section{CONCLUSIONS:}

Our study demonstrates that stable micron- and nano-size emulsions, liposomes, and aqueous suspensions can be produced using both "top down" and "bottom up" methods. The formulation properties, in terms of particle size and stability, strongly depend on the processing parameters used in terms of energy input and temperature history. The energy requirements of the "bottom up" methods may be substantially lower than those of "top down" methods. The dimensions of the micro-channels within the impinging jet devices, the fluid velocity, and the temperature history during processing were varied and optimized for each formulation. Thus, the results presented here, with particular focus on stability, bioavailability, and consumer acceptance, confirm that the platform technologies and the respective operational maps presented here are suitable for the production of functional food formulations with micron-and nano- size specifications. Unfortunately, there still remain some barriers to successful implementation of cost effective manufacturing processes for the final macro-scale products. These remaining challenges rest mainly with the incorporation of these specially formulated nutraceutical ingredients into a large scale manufacturing system; mechanical stability and related shelf-life issues are major concerns. Fortunately, as demonstrated here, proper processing of these 
nutraceutical and functionalized food formulations can help in overcoming many of these challenges, and subsequently meet a major global objective of socially responsible food companies. That is, they strive to provide improved quality foods, enriched with ingredients that are in limited supply in our food chain; to enhance human health and wellness world-wide.

Competing Interests: The authors have no financial interests or conflicts of interest.

Authors' Contributions: All authors contributed to this study.

Acknowledgements and Funding: The authors would like to thank Mr. Kenneth Chomistek for conducting the curcumin experiments and Microfluidics International for support.

\section{REFERENCES:}

1. Berdanier C. Advanced Nutrition Micronutrients. 1997; CRC Press ISBN 0849326648, pp 27-9

2. Chow CK. Fatty Acids in Food and their Health Implications. 2001; Routledge Publ. New York, NY

3. Merisko-Liversidge E, Liversidge G, Cooper E. Nanosizing: A Formulation Approach For Poorly-Water-Soluble Compounds. Eur. J. Pharm. Sci. 2003; 18:113-123.

4. Rabinow BE. Nanosuspensions in Drug Delivery. Nature Reviews Drug Discovery 2004; 3(9): 785-796.

5. Rabinow BE. Pharmacokinetics of Nanosuspensions, Proceedings of the Nanotechnology for Drug Delivery Conference 2005; Philadelphia, Pa, USA.

6. Panagiotou T, Fisher RJ. Form Nano-Particles Via Controlled Crystallization: A“BottomUp" Approach. Chem. Eng. Prog. 2008; 104(10): 33-39.

7. Panagiotou T, Mesite S, Fisher RJ. Production Of Norfloxacin Nano-Suspensions Using Microfluidics Reaction Technology (MRT) Through Solvent/Anti-Solvent Crystallization. Ind. Eng. Chem. Res. 2009; 48(4): 1761-1771.

8. Liversidge GG. Controlled Release And Nanotechnologies: Recent Advances And Future Opportunities. Drug Development and Delivery 2011; 11: 1-7.

9. Panagiotou T, Fisher RJ. Enhanced Transport Capabilities via Nanotechnologies: Impacting Bio-efficacy, Controlled Release Strategies and Novel Chaperones. J. Drug Delivery 2011; ID 902403, 1-14.

10. Panagiotou T, Fisher RJ. Improving Product Quality with Entrapped Stable Emulsions: From Theory to Industrial Application. Challenges 2012; Vol. 3: 1-39.

11. Okushima S, Nisisako T, Torii T, Higuchi T. Controlled Production Of Monodisperse Double Emulsions by Two Step Droplet Breakup in Microfluidic Devices. Langmuir 2004; 20: 9905-9908.

12. Hakansson A, Innings F, Tragardh C, Bergenstahl B. A High-Pressure Homogenization Emulsification Model-Improved Emulsifier Transport And Hydrodynamic Coupling. Ch Eng Sci 2013; 91: 44-53.

13. Lasic DD. Liposomes In Gene Delivery. 1997 CRC Press LLC, Boca Raton, FL. 
14. Drusch $\mathrm{S}$, et al. Stabilization of Omega-3 fatty Acids by Microencapsulation. Agro FOOD 2008;19(4): 31-32.

15. Mozaffarain D, Rimm EB. Fish Intake, Contaminates, and Human Health: Evaluating the Risks and Benefits. JAMA 2000; 296(15): 1885-1899.

16. Huang Q. Micro/nanoencapsulation of Active Food Ingredients. American Chemical Society, proceedings of ACS Symposium Series XIV, Washington, DC, 2009; 314(1): 8.

17. Soottitantawat A. Microencapsulation by Spray Drying: Influence of Emulsion Size on the Retention of Volatile Compounds. Journal of Food Science 2003; 68(7): 2256-2262.

18. Hogan SA. Microencapsulating Properties of Sodium Caseinate. Journal of Agricultural and Food Chemistry 2001; 49(4): 1934-1938.

19. Ma Y, Reineccius GA. The Stability of Spray-Dried Microcapsules as a Function of Glass Transition Temperature. Proceedings of the Institute of Food Technologists Annual Meeting, New Orleans, LA, June 1992; 17-18.

20. Kjaergaard OG. Multiple-Core Encapsulation. Microencapsulation of Food Ingredients, VP, Editor, Leatherhead, Surrey, UK, 2001; 133-144.

21. Keogh MK. Stability to Oxidation of Spray-Dried Fish Oil Powder Microencapsulated Using Milk Ingredients. J. Food Sci. 2001; 66(2): 217-224.

22. Drusch S, Serfert Y, Schwarz K. Microencapsulation of Fish Oil with NOctenylsuccinate-Derivatised Starch: Flow Properties and Oxidative Stability. European Journal of Lipid Science and Technology 2006; 108(6):501-512.

23. Miller MT. In Vitro Evaluation of Cytotoxicity and Cellular Uptake of Alternating Copolymers for use as Drug Delivery Vehicles. Ph.D. Dissertation, Department of Chemical Engineering, Massachusetts Institute of Technology, June 2009.

24. Gomez-Hens A, Fernandez-Romero JM. Analytical Methods For The Control of Liposomal Delivery Systems. Trends Anal Chem 2006; 25: 167-178.

25. Mozafari MR, Johnson C, Hatziantoniou S, Demetzos C. Nanoliposomes And Their Applications In Food Nanotechnology. J. Liposome Res. 2008; 18(4): 309-327.

26. Mozafari MR, Mortazavi SM. Nanoliposomes: From Fundamentals To Recent Developments. 2005; Trafford Publishing Ltd, Oxford, UK.

27. Bender DA. Nutritional Biochemistry Of Vitamins 2003; Cambridge, UK.

28. Bowen PE, Herbst-Espinosa SM, Hussain EA, Stacewicz-Sapuntzakis M. Esterification Does Not Impair Lutein Bioavailability In Humans. J. Nutr. 2002; 132(12) 3668-3673.

29. Johnson EJ, Hammond BR, Yeum KJ. Relation Among Serum And Tissue Concentrations Of Lutein Andzeaxanthin And Macular Pigment Density. Am J. Clin Nutr. 2000; 71(6): 1555-62.

30. Johnson EJ, Neuringer M, Russell RM, Schalch W, Snodderly DM. Nutritional Manipulation Of Primate Retinas, III: Effects Of Lutein Or Zeaxanthin Supplementation on Adipose Tissue And Retina Of Xanthophyll-Free Monkeys. Invest. Ophthalmol. Vis. Sci. 2005; 46(2): 692-702.

31. Richer S, Stiles W, Statkute L. Double-Masked, Placebo-Controlled, Randomized Trial of Lutein And Antioxidant Supplementation. Optometry 2004; 75(4): 216-30. 
32. SanGiovanni JP, Chew EY, Clemons TE. The Relationship of Dietary Carotenoid and Vitamin A, E, and C Intake with Age-Related Macular Degeneration in a Case-Control Study: AREDS Report No. 22. Arch. Ophthalmol. 2007; 125(9): 1225-1232.

33. Baker FM. Dietary Supplementation: Effects nn Visual Performance and Occurance of Amd And Cataracts. Current Medi. Res. and Opinion 2010; 26(8): 2011-2023.

34. Lantz RC, Chen GJ, Solyom AM, Jolad SD, Timmerman BN. The Effect of Turmeric Extracts on Inflammatory Mediator Production. Phytomedicine 2005; 12: 445-452.

35. Kunchandy E, Rao, MNA. Oxygen Radical Scavenging Activity Of Curcumin. Int. J. Pharm. 1990; 58: 237-240.

36. Sharma RA, Gescher AJ, Steward WP. Curcumin: The Story So Far. Eur. J. Cancer 2005; 14: 1955-1968.

37. Khanna NM. Turmeric: Nature's Precious Gift. Curr. Sci. 1999; 76: 1351-1356.

38. Ringman JM, Frautschy SA, Cole GM, Masterman DL, Cummings JL. A Potential Role of the Curry Spice Curcumin in Alzheimer's Disease. Curr Alzheimer Res. 2005; 2: 131- 136.

39. Funk JL, Oyarzo JN, Frye JB, Chen G, Lantz RC, Jolad SD, Solyom AM, Timmerman BN. Turmeric Extracts Containing Curcuminoids Prevent Experimental Rheumatoid Arthritis. J. Nat. Prod. 2006; 69: 351-355.

40. Donsi F, Wang Y, Li J, Huang Q. Preparation of Curcumin Sub-micrometer Dispersions by High-Pressure Homogenization. J. Agric. Food Chem. 2010; 58: 2848-2853.

41. Onque S, Takahashi H, Kawabata Y, Seto Y, Hatanaka J, Timmerman B, Yamada S. Formulation Design And Photochemical Studies On Nanocrystal Solid Dispersion of Curcumin With Improved Oral Bioavailability. J. Pharma. Sci. 2010; 99(4): 1871-1881.

42. Maiti K, Mukherjee K, Gantait A, Saha BP, Mukherjee PK. Curcumin-phospholipid Complex: Preparation, Therapeutic Evaluation and Pharmacokinetic Study In Rats. Int. J. Pharm. 2007; 330: 155-163.

43. Sharma RA, Gescher AJ, Steward WP. Curcumin: The Story So Far. Eur. J. Cancer 2005; 41: 1961-1969.

44. Tonnesen HH, Karlsen J, van Henegouwen GB. Chemistry of Curcumin and Curcuminoids. Z. Lebensm Unters Forsch 1986; 183: 116-122.

45. Chen C, Johnston TD, Jeon H, Gedaly R, McHugh PP, Burke TG, Ranjan D. An In Vitro Study of Liposomal Curcumin: Stability, Toxicity And Biological Activity In Human Lymphocytes And Epstein-Barr Virus-Transformed Human B-Cells. Int. J. Pharm. 2009; 366: 133-139.

46. Tonnesen HH, Masson M, Loftsson T. Studies Of Curcumin and Curcuminoids. XXVII. Cyclodextrin Complexation: Solubility, Chemical And Photochemical Stability. Int. J.Pharm. 2002; 244: 127-135.

47. Tomren MA, Masson M, Loftsson T, Tonnesen HH. Studies on Curcumin and Curcuminoids. XXXI. Symmetric and Asymmetric Curcuminoids: Stability, Activity and Complexation with Cyclodextrin. Int. J. Pharm. 2007; 338: 27-34.

48. Letchford K, Liggins R, Burt H. Solubilization of Hydrophobic Drugs by Methoxy Poly(Ethylene Glycol)-Block-Polycaprolactone Diblock Copolymer Micelles: Theoretical and Experimental Data And Correlations. J. Pharma. Sci. 2008; 97: 1179-1190. 
49. Ravindranath V, Chandrasekhara N. Absorption and Tissue Distribution of Curcumin in Rats. Toxicology 1980; 16: 259-265.

50. Cheng A, Hsu CH, Lin JK, Hsu MM, Ho YF, Shen TS, Ko JY, Lin JT, Wu MS, Yu HS, Jee SH, Chen GS, Chen TM, Chen CA, Lai MK, Pu YS, Pan MH, Wang YJ, Tsai CC, Hsieh CY. Phase I Clinical Trial of Curcumin, a Chemopreventive Agent, in Patients with High-Risk or Pre-Malignant Lesions. Anticancer Res. 2001; 21: 2895-2900.

51. Pan MH, Huang TM, Lin JK. Biotransformation Of Curcumin Through Reduction and Glucuronidation in Mice. Drug Metab. Dispos. 1999; 27: 486-494.

52. He Y, Huang Y, Cheng Y. Structure Evolution of Curcumin Nanoprecipitation from a Micromixer. Crystal Growth and Design/Comm. 2010; 10: 1021-1027.

53. US Patent No. 8187554; Apparatus and Methods for Nano-Particle Generation and Process Intensification of Transport and Reaction Systems. May 2012. 